\title{
The wicking characteristics of a sustained release delivery system for the inner ear: the silverstein micro wick
}

\begin{abstract}
Objective: To explore the properties of the Silverstein Microwick ${ }^{\circledR}$

Background: Direct drug delivery to the inner ear via the round window membrane is becoming increasingly common. The Silverstein Microwick is an example of a sustained release delivery system. Medication regimens for this wick have largely been chosen arbitrarily as there is no data relating to its inner ear kinetic profile and no knowledge of its wicking behavior.
\end{abstract}

Methods: A custom-built device was used to measure electrical impedances obtained from a series of 6 electrodes inserted into a Silverstein Microwick in order to define the time course of how different parts of the wick dry out. The impedances across adjoining pairs of electrodes were used to measure the "wetness" of each segment. Three drops of gentamicin solution were applied. We sought to determine the flow of the solutions through the wick by analyzing changes in electrical impedance. Various medications were applied to the wick including dexamethasone. Other properties inherent to the wick were also measured including drug availability at the round window membrane end and its filtering capacity.

Results: It was determined that the wick was completely dry after 105-110minutes and that it dried faster at its extremities. The electrical impedance of the rwm end of the wick reached its pre-test level after 100-105minutes. However, drug availability at the rwm end of the wick ceased after 70-75minutes. Adding a further 3 drops after 1hour prolonged the drying time by 100 minutes. A $15 \%$ elevation in environmental humidity resulted in a drying time of 400minutes. Liquid suspensions were not ideal for use through the wick as components of suspensions are retained by the wick material. Suspensions were also found to have the highest viscosities.

Conclusion: The most therapeutic inner ear profile for treatment of Meniere's is unknown. Although pumps can be used to generate any kind of profile, they are not currently licensed for use in humans and are invasive and expensive. The Silverstein microwick has been used successfully in humans and is non-invasive and cheap. Only inner ear studies will be able to determine their exact inner ear kinetic profile associated with this device. However, the profiles achievable with this device will largely be determined and ultimately limited by the behavior/characteristics of the wick. Our study sheds light on this. Given that drug availability to the round window membrane is $70-75$ minutes we can surmise the most likely inner ear kinetic profile that would ensue. The dosing regimen for the microwick could be altered to influence this. Inner ear studies are required to determine if changes in wick dosing parallel events in the inner ear.

Keywords: wick, round window, sustained release, transtympanic injection
Volume 3 Issue 3 - 2015

Bance M, Kaushik V, Fowler S, Khwaja A, Van

Wijhe R

EAR Laboratory, Dalhousie University, Canada

Correspondence: Bance M, EAR Laboratory, Dalhousie University, 3184 Dickson Building,VGH Site, 1278 Tower Road, Halifax, NS, Canada, Email mbance@dal.ca

Received: October 27, 2014 | Published: September 23, 2015

\section{Introduction}

Local application of drugs to the inner ear has become a popular method to treat a variety of disorders of the inner ear, including Meniere's disease, sudden sensorineural hearing loss, autoimmune inner ear disease and tinnitus. The affected ear can be targeted precisely and in higher concentration, without the need for systemic administration and its associated side-effects. Many of the methods available enable this to be achieved within the convenience of the Otolaryngologist's office. There are several commonly practiced techniques of round window membrane perfusion, which include transtympanic injection into the middle ear space, injection onto Gelfoam (Upjohn, Kalamazoo, MI) placed in the round window niche and sustained release devices such as the Round Window Microcatheter (Durect Corp., Cupertino, CA) and the Silverstein MicroWick (Micromedics, Eaton, MN).
The level of vestibular symptom control achieved with transtympanic injection of gentamicin into the middle ear for Meniere's disease has been extremely varied. It is thought that this inconsistency is due to variable amounts of medication escaping down the Eustachian tube and medication filling the middle ear space but not being in contact with the round window membrane where the vast majority of absorption occurs. Transtympanic injection is associated with high peak concentrations in the inner ear which may be detrimental by way of ototoxicity. Attempts have been made to stabilize the applied volume using hyaluronic acid and resorbable gelatin sponges, although none have proved popular in clinical use.

In comparison, devices that deliver medication directly to the round window membrane permit a controlled amount of medication to be applied to the round window membrane allowing the flexibility of adjusting delivery. Consequently, investigators can reliably and 
consistently manipulate inner ear kinetic curves. ${ }^{1}$ It has not yet been established whether a rapid peak with short duration is preferable to a more sustained steady state profile. ${ }^{2}$ It has been theorized that toxicity in the inner ear may be a dose-dependent phenomena with higher levels resulting in profound hearing loss. ${ }^{3}$ Consequently, lower initial peak of gentamicin concentrations in the inner ear may widen the therapeutic window/index between intentional vestibular damage and inadvertent cochleotoxicity. ${ }^{4}$ In addition, these devices allow formulations to be provided for extended times without the need for repeat visits to the physician's office and it permit clinicians to make more meaningful cross-study comparisons.

To our knowledge, the only direct round window membrane delivery device currently available for use in humans is the Silverstein Microwick ${ }^{\mathrm{TM}}$ (Micromedics, MN, USA). Production of the human round window microcatheter $\left(\mu-\mathrm{Cath}^{\mathrm{TM}}\right)$ (Durect) ceased in 2003 due to a change in business focus. This system was more expensive compared with the Silverstein microwick and required placement under a general anaesthetic. The Alzet $\AA$ osmotic pump produced by the same company is only available for animal use.

The Silverstein Microwick is made of a polyvinyl acetate material and measures (when dry) $1 \mathrm{~mm}$ by $9 \mathrm{~mm}$. It can be inserted under local anesthetic in a non-certified office surgery room, is cheap and appears to be well tolerated whilst in-situ. Insertion involves performing a tympanotomy over an estimated position of the round window niche. A ventilation tube is inserted through the tympanotomy and a wick is inserted into the tube, with its tip placed into the round window niche. Medication drops are placed onto the Microwick and it is allowed to expand. The patient then self-administers medication to the ear canal. When the medication contacts the proximal end of the Microwick it becomes saturated with the medication and the medication travels to distal end under capillary action. As distal end is in contact with round window membrane, the medication permeates the round window and is absorbed into the inner ear fluids.

Although the inner ear kinetic profile of the microwick has yet to be defined, the microwick, when perfused with gentamicin, has been shown to be an effective method of treating disabling vertigo associated with Meniere's disease ${ }^{5}$ (Jackson $^{5}$ Chemical perfusion of the inner ear). A typical administration protocol requires three drops of dilute Gentamicin otic solution ( 5 to $10 \mathrm{mg} / \mathrm{ml}$ ), three times daily per day for one to three weeks for treatment of Meniere's disease and dexamethasone solution $(24 \mathrm{mg} / \mathrm{cc}$ three times daily for several weeks) for treatment of sudden deafness or autoimmune ear disease.

At this point in time it is not known how long each dose maintains saturation of the microwick and more importantly how long it sustains delivery of the drug to the round window membrane. This study aims to quantify the wicking behavior of the Silverstein microwick and add to the knowledge base of this delivery system. It is hoped that our findings will help inform clinicians of the kinetic profile likely to be imparted to the inner ear by a given dosing regimen. It may also prove useful to investigators design future trials studying inner ear kinetic curves associated with the device. Ultimately, once the most therapeutic inner ear profile has been identified, we will be able to work backwards and determine the microwick dosing schedule that will achieve this (Figure 1).

\section{Materials and methods}

We chose to measure electrical impedance as a surrogate measure of the wetness of the wick for a number of reasons. Firstly, it allowed us to measure the drying time of different portions of the wick so that we could determine how it dries, rather than just measuring weight which would only tell us total drying time. Secondly, it was more stable and less fluctuant than the very small weight changes that were observed during drying.

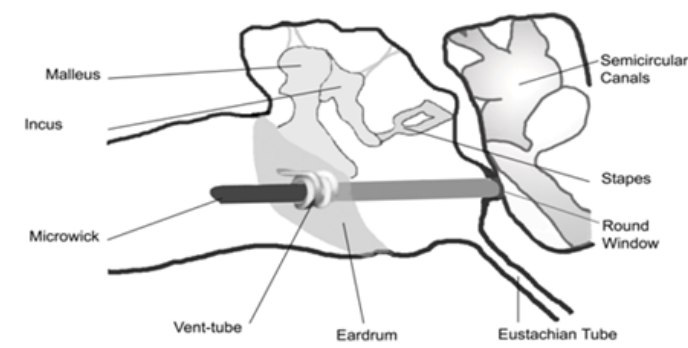

Figure I Drawing showing the Microwick placement against the round window membrane.

Experiment 1: Measuring the electrical impedance of a drying microwick.

Objectives: To determine how long it takes for the wick to dry and whether it dries evenly.

Setup and Procedure: The resistance across six points on the microwick was measured to determine how the microwick dries. The tests were performed in an environment similar to that of the ear canal, which was simulated within a Styrofoam cooler. A 150watt light bulb with a dimmer switch was suspended from one end of the enclosure, which could be adjusted to provide a temperature of $37 \pm 2$ degrees. An opening was made next to that of the light bulb and a piece of plastic tubing was inserted connected to a humidifier that maintained a humidity of $70 \% \pm 4 \%$. The temperature dial of the humidifier (Fisher \& Paykel Healthcare, HC 150) could be adjusted according to changes in the enclosure and laboratory environment. These conditions were monitored by a temperature sensor and a humidity sensor placed adjacent to the Microwick.

The resistance device consisted of six electrodes that are fixed to one end of a rectangular piece of rigid foam and protrude from it. This device was modified from its original configuration which had the Microwick sitting on top of the electrical contacts. It was found that moisture from the wick could track down and short adjacent contacts, which did not happen once we reversed the placement and put the contacts on top. All earlier results were discarded. The foam slides vertically into a pair of grooved pillars and is lowered until the electrodes make contact lengthwise with the microwick sitting on a stiff plastic platform connected to the vertical pillars. Five areas on the microwick's surface are measured using the six electrodes. The electrodes are arranged in parallel with six $33 \mathrm{~K}$ resistors and through an interface, connected to a terminal block for thermocouples (National Instruments, TBX-68T). The terminal block sends analog signals to an internal board and a custom-designed Lab view program displays readings of resistance, temperature, humidity and weight. The weight of a drying microwick over time was measured alongside the resistance readings to enhance validity and will be discussed later. For the purposes of analysis, the five segments of the wick being monitored during the experiment were referred to by the numbers 1 to 5. The end of the wick that would be protruding into the ear canal and to which medication would be applied was deemed to be segment 1 . The segment of the wick which would be next to the round window membrane in vivo was referred to as segment 5 . 
Three drops of gentamicin otic solution (Schering) was applied to segment 1of a new microwick. The wick expands slightly upon introduction of the liquid. The Lab view program is initiated and the device is placed into the environment, which is stable at the desired environmental conditions. The resistance is allowed to rise until it reaches its original dry state value of 32kilo ohms. Our assessment intervals were 20 seconds. We also measured the results after the first 10seconds. After each experiment, the wicks were rinsed thoroughly with distilled water and allowed to dry completely before they could be used for further tests. Figure 2 shows a summary of thirty-five resistance trials.

Experiment 2: Validity tests.

\section{Weight tests}

Rationale: The ideal apparatus setup would allow us to measure the weight of the microwick at the same time as being able to measure its electrical impedance. This would allow us to directly correlate electrical impedance with drug volume applied to the wick and validate that the device was measuring wetness. However, difficulties encountered with stability of the electronic balance and electrode setup meant that this was not possible, even after modifying the setup. Consequently we were forced to measure these parameters independently. The weight-time and electrical impedance-time data were then used to plot weight against electrical impedance.

Procedure: A dry microwick was placed on a plastic dish and positioned inside the glass enclosure of a sensitive electronic balance (Denver Instruments APX-60). The mass of this dry setup was noted. Then, three drops of medication were applied to the microwick and the Lab view program initialized and measurements taken every 20 seconds. When the wet mass had decreased to the dry wick mass, the test was stopped. The experiment was repeated several times. The time course for this weight change was compared to the time course for electrical impedance change. Variations on the original experiment were made to further verify if electrical impedance could be used as a surrogate measure of drug volume applied to the wick and of drying time.

\section{Environmental tests}

Rationale: Environmental changes that alter dry time should also cause a corresponding change in electrical impedance with time.

Procedures: The humidity was set approximately $10 \%$ higher than during the original tests. The ambient temperature remained the same. Using a heat source: Several trials were conducted to establish how long it took for the microwick to dry in the laboratory environment. The trials were repeated whilst a 1600 watt hairdryer was blown onto the microwick.

\section{Interventional tests}

Rationale: Interventions that change that change dry time should also cause a corresponding change in electrical impedance with time.

Procedure: Adding drops over time: A standard trial was run. After 60 minutes, three additional drops were added to the ear canal end of the wick.

Experiment 3: Drug availability at the round window membrane end of the microwick.

Objectives: To determine approximately how long applied medication is available at the round window membrane end of the microwick.
Setup and Procedure: The basic resistance test was run. When segment 5 of the microwick reached a resistance of $8 \mathrm{~K} \mathrm{ohms} \mathrm{a} \mathrm{piece}$ of filter paper (Fisher Scientific P8), with a known mass, was held to one end. The filter paper was held by a clamp on a stand. The pressure applied was slight, so that the Microwick was just touching the filter paper. The filter paper was allowed to absorb liquid from the Microwick for 30seconds, after which the filter paper was immediately weighed to avoid further evaporation. The weight of medication transferred to the filter paper was then calculated. This value was akin to the drug availability at the rwm end of the wick. This constituted one trial. A new trial, using a new wick, was commenced but this time the availability at $10 \mathrm{~K}$ ohms was calculated. Further trials at successive $2 \mathrm{k}$ ohms increments were conducted until a point was reached were no fluid was imparted onto the filter paper. We were therefore able to map out drug availability at the rwm end of the microwick at various segment 5 electrical impedances. In addition to testing the behavior of the microwick, we ran several tests on liquid medications used through the microwick.

Experiment 4: Analyzing the filtering capacity of the Microwick.

Objective: To determine if the Microwick filters out any medication components.

Setup and Procedure: Liquid chromatography-mass spectrometry is an analytical chemistry technique that combines the physical separation capabilities of liquid chromatography with the mass analysis capabilities of mass spectrometry. For this experiment four solutions were passed through the Microwick and analysed. They were as follows:

1. Methylprednisolone Acetate Injectable Suspension USP, 40mg/ $\mathrm{mL}$, Sabex ${ }^{\circledR}$, lot\#115920

2. Dexamethasone Sodium Phosphate Injection USP, $4 \mathrm{mg} / \mathrm{mL}$, Sabex ${ }^{\circledR}$, lot\#116143

3. Garasone Ophthalmic/Otic Solution/Topical Corticosteroid Antibiotic, 3mg/ml Gentamicin, Schering ${ }^{\circledR}$, lot\#01A22 02B

4. CiproHC Otic Suspension, $0.2 \%$ Ciprofloxacin, $1 \%$ Hydrocortisone, Alcon ${ }^{\circledR}$, lot $\# 263083 \mathrm{BB}$

5. SoluMedrol, Methylprednisolone Sodium Succinate Sterile Powder (Methylprednisolone Sodium Succinate for Injection, USP), $125 \mathrm{mg}$ Act-O-Vial () System (Single-Dose Vial), Pharmacia \& Upjohn Company.

Six $1 \mathrm{ml}$ containers with attached lids were weighed, labeled and their masses recorded. A new Microwick was inserted into a plastic sheath which had a diameter of $2 \mathrm{~mm}$. The Microwick and sheath were held over an open container and a needle used to apply the solution onto the sheath. The medication was allowed to pass through the Microwick. It was collected with a $1 \mathrm{~mm}$ diameter glass capillary tube, of known weight and the tube containing the filtered medication was deposited into the container. The cover was applied. The container was re-weighed. The mass of the sample could then be calculated. This process was repeated five times. The samples were taken to our University Chemistry Department. The samples were diluted, exactly the same amount with methanol, to enable the mass spectrometer to process them. Then $10 \mu \mathrm{L}$ of each sample was run through the mass spectrometer and a mass chromatogram was produced. Six mass chromatograms of the pure, unfiltered medication were also produced.

Experiment 5: Measurement of Viscosity. 
The viscosity of different medications at body temperature was determined, as viscosity is inversely related to capillary action.

Objective: To measure the viscosity of medications used through the Microwick.

Rationale: Viscosity describes a fluid's internal resistance to flow. Theoretically a solution with a lower viscosity will have an increased flow rate.

Setup and Procedure: The medications used in this experiment are all commonly prescribed for inner ear conditions and were as follows:

1. Methylprednisolone Acetate Injectable Suspension USP, $40 \mathrm{mg} /$ $\mathrm{mL}$, Sabex ${ }^{\circledR}$, lot $\# 115920$

2. Dexamethasone Sodium Phosphate Injection USP, $4 \mathrm{mg} / \mathrm{mL}$, Sabex ${ }^{\circledR}$, lot\#116143

3. Dexamethasone Sodium Phosphate Ophthalmic Solution USP, $1 \mathrm{mg} / \mathrm{mL}$, Sabex ${ }^{\circledR}$, lot $\# 116941$

4. Garasone Ophthalmic/Otic Solution/Topical Corticosteroid Antibiotic, 3mgGentamicin/mL, Schering ${ }^{\circledR}$, lot\#01A22 02B.

The viscosities of all solutions except Methylprednisolone suspension were tested using the Viscomat (Pfeuffer (C)). The Viscomat measures the time necessary for a defined volume of liquid to pass through a capillary at a known differential and defined temperature. The resulting viscosity is indicated in millipascal-seconds (mPas). The Viscomat was calibrated with distilled water at $37^{\circ} \mathrm{C}$ and all readings were determined at the same temperature.

The viscosity of the Methylprednisolone suspension was determined using the Bohlin VOR rheometer. The Bohlin works using a cone and plate assembly. The cone is elevated $1^{\circ}$ from the horizontal and to the plate is added $5 \mathrm{~mL}$ of the liquid to be tested. The plate spins and a sensor on the cone measures torque, which is proportional to shear stress. The rate of rotation is proportional to the shear rate. Shear stress divided by shear rate gives viscosity in $\mathrm{mPas}$. The Bohlin was also calibrated at $37^{\circ} \mathrm{C}$

\section{Results}

\section{First experiment}

See Figure 2. The maximum resistance is reached by all parts of the Microwick at approximately 105-110minutes. At this point in the drying process the Microwick is reduced to its original size and is dry and "crispy" to the touch. At $85 \%$ humidity, the Microwick took about 400 minutes to reach $33 \mathrm{kilo}$ ohms. This is over 3.5 times as long as the control experiment at $70 \%$ humidity. By way of comparison, the humidity within the ear canal of a human subject measured with our device was $70 \%$. Based on electrical impedance, the laboratory dry time for the microwick was determined to be approximately 70 minutes. After application of the heat source the wick became completely dry after only 7 minutes. Adding 3 drops to the microwick after one hour increased the drying time by 100 minutes.

\section{Second experiment}

The 7 weight trials showed that the Microwick dried after approximately 105-110minutes (Figure 3).The relationship between weight and electrical impedance was found to be linear $\left(\mathrm{P}=.000, \mathrm{R}^{2}=\right.$ 0.9553) (Figure 4).

\section{Third experiment}

Mean drug availability at RW membrane end of wick (Table 1) (Figure 5).

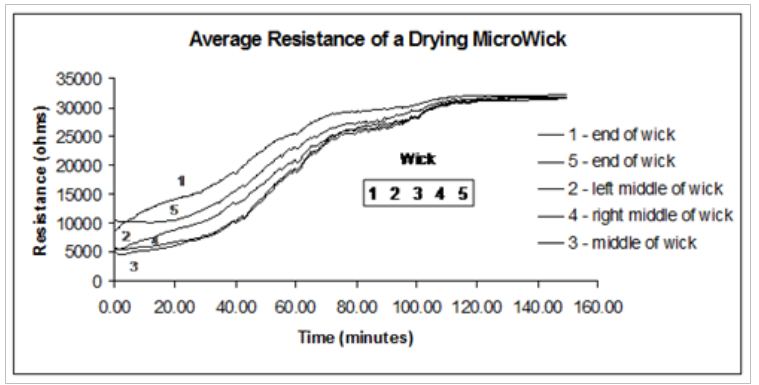

Figure 2 Average resistance of different parts of the drying wick.

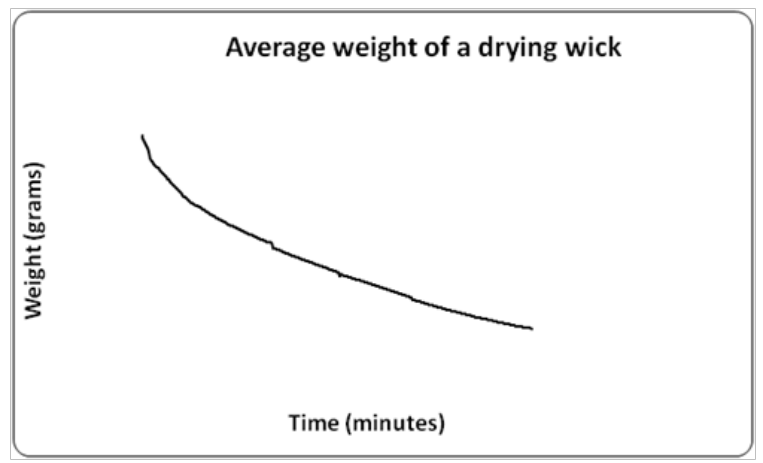

Figure 3 Resistance versus weight.

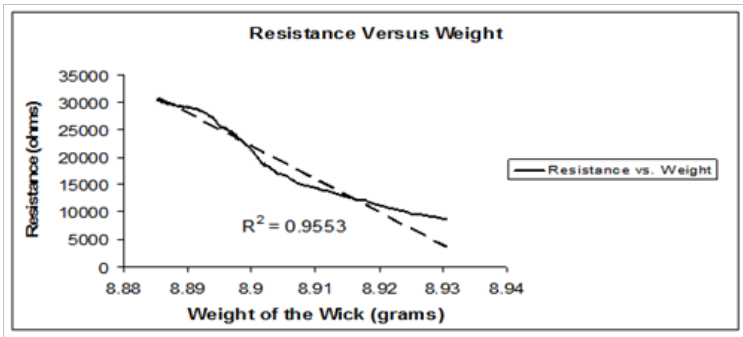

Figure 4 The relationship between weight and electrical impedance was found to be linear $\left(P=.000, R^{2}=0.9553\right)$.

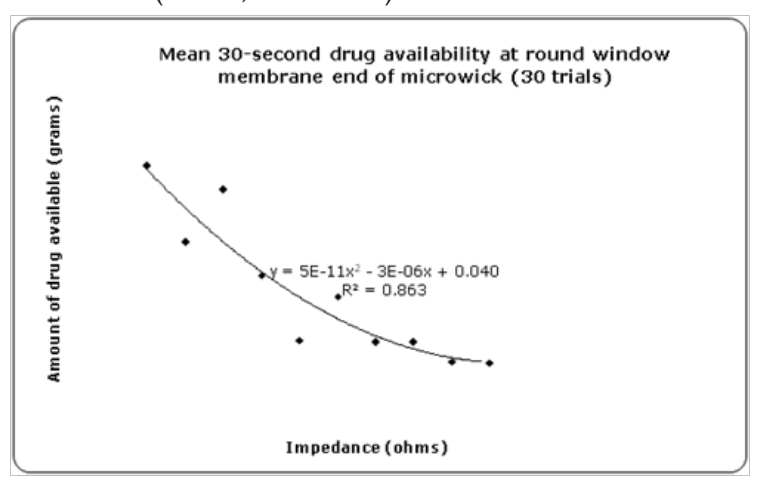

Figure 5 Mean drug availability at RW membrane end of wick.

\section{Fouth experiment}

A mass spectrum represents a method of chemically analyzing a sample. It is usually acquired using an instrument called a mass spectrometer. It is a plot of intensity versus mass-to-charge ratio. The pattern displayed represents the distribution of components (atoms or molecules) by mass (more correctly: mass-to-charge ratio) in a sample (Figure 6).

A chromatogram is the visual output of the chromatograph. Each peak corresponds to different components within the sample 
(Figure 7). The higher the peak, the more component is present. For the Garasone experiment, the highest peak was one of the gentamicin components. There were four other much smaller peaks that represented other gentamicins, betamethasone and benzalkonium chloride (preservative). In order to determine the amount of drug before and after the Microwick, the area under all of the peaks was calculated. Table 2 shows the mean area with standard deviation for each sample before and after the Microwick. The percentage difference was also calculated.
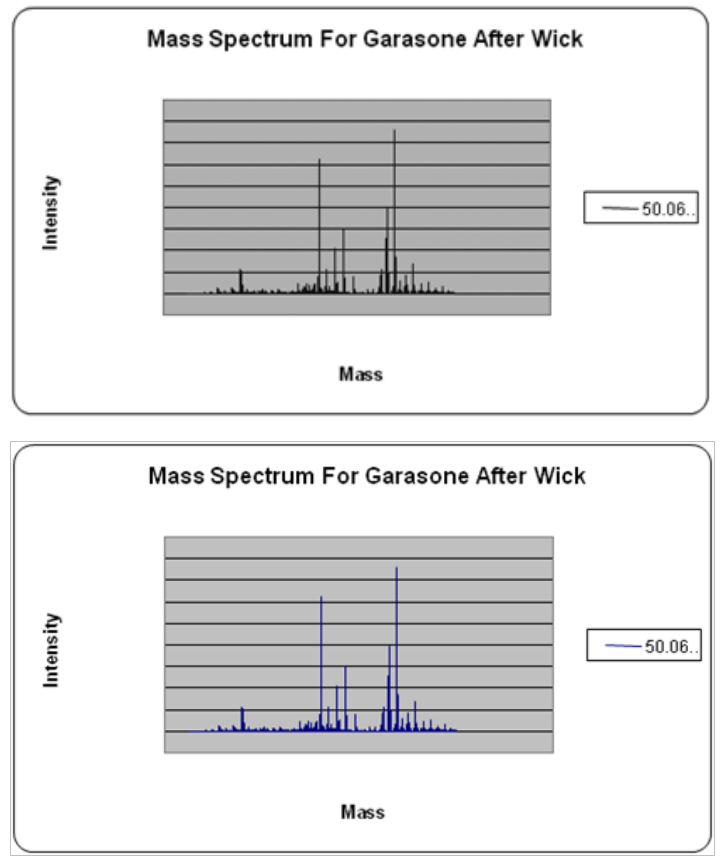

Figure 6 Mass spectrometry of fluid before and after filtering by wick.
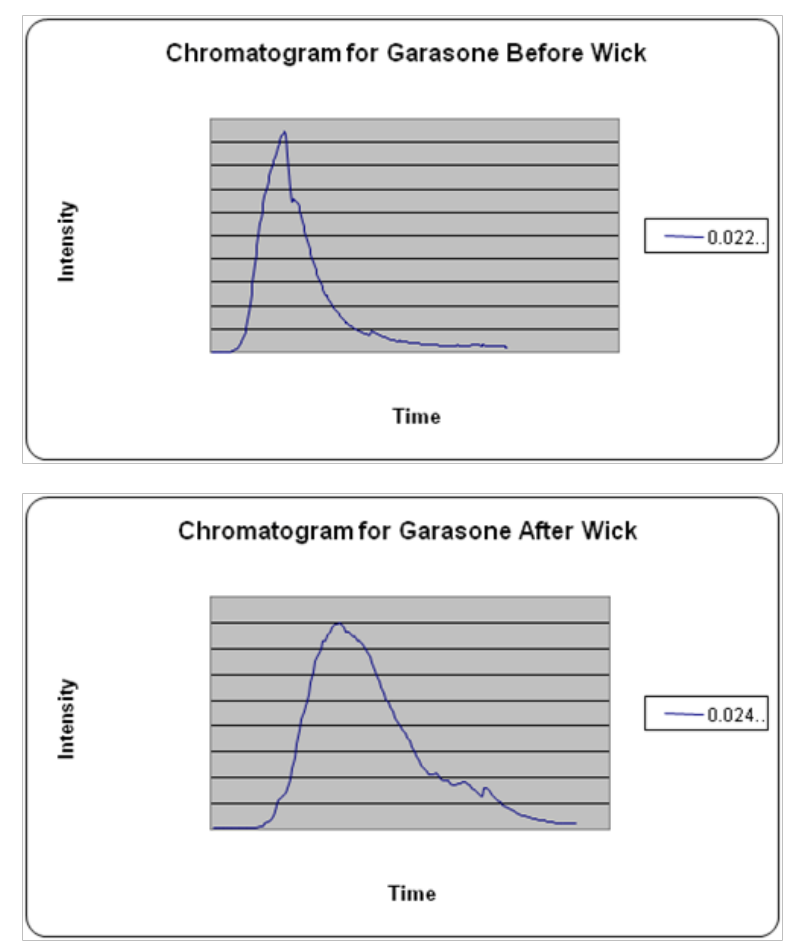

Figure 7 Chromatography of Garasone before and after filtering by wick.

\section{Fifth experiment}

Viscosities with error calculations of five commonly prescribed inner ear medication. Normalized Values - The viscometers were both calibrated with water at $37{ }^{\circ} \mathrm{C}$ and compared to the $\mathrm{CRC}$ value for water. The CRC value for water was found to be $81 \%$ of the value determined by the Viscomat; therefore all the viscosities were multiplied by 0.81 , yielding the normalized values. The normalizing ratio for the Bohlin VOR was 1.03 (Table 3).

Table I Mean drug availability at RW membrane end of wick

\begin{tabular}{|c|c|c|c|c|}
\hline \multirow[t]{3}{*}{$\begin{array}{l}\text { Electrical } \\
\text { Impedance } \\
\text { at Segment } 5 \\
\text { (Kilo Ohms) }\end{array}$} & \multicolumn{3}{|c|}{$\begin{array}{l}\text { Amount Of Drug Available at } \\
\text { Round Window Membrane End } \\
\text { Of Microwick (Grams) }\end{array}$} & \multirow[t]{2}{*}{$\begin{array}{l}\text { Mean Drug } \\
\text { Availability } \\
\text { (Grams) }\end{array}$} \\
\hline & \multicolumn{3}{|c|}{ (Individual Trial Results Shown) } & \\
\hline & Trial I & Trial 2 & Trial 3 & \\
\hline 8 & 0.0202 & 0.0193 & 0.022 & 0.0205 \\
\hline 10 & 0.0168 & 0.0093 & 0.0115 & 0.0125 \\
\hline 12 & 0.0209 & 0.0247 & 0.0083 & 0.018 \\
\hline 14 & 0.0074 & 0.0143 & 0.0056 & 0.0091 \\
\hline 16 & 0.0016 & 0.0042 & 0.0012 & 0.0023 \\
\hline 18 & 0.0045 & 0.0108 & 0.0054 & 0.0069 \\
\hline 20 & $0.004 I$ & 0.0002 & 0.0022 & 0.0022 \\
\hline 22 & 0.0014 & 0.0037 & 0.0013 & 0.0021 \\
\hline 24 & 0 & 0.0004 & 0 & $0.000 \mathrm{I}$ \\
\hline 26 & 0 & 0 & 0 & 0 \\
\hline
\end{tabular}

\section{Discussion}

\section{First experiment}

The results from the electrical impedance test show that under normal physiological conditions the drying curve is sigmoid in shape with most of the drying process occurring between 40-70minutes (Figure 2). Prior to the application of the drops, impedances from all segments were in excess of $3000 \mathrm{ohms}$ (not shown on graph). Our first measurement of electrical impedance was performed at 10 seconds. One would expect segment 1 to have the lowest impedance as medication was applied to this section of the wick. This was not observed. In fact, at 10 seconds, the central portions of the wick were most wet, followed by the application end. The membrane end was least wet. The most likely explanation is that our measurement intervals missed the period of spontaneous drug flow driven by capillary forces (wicking) that occurred when the medication was first applied; by the time our first measurement was taken, at 10seconds, the bulk of the travelling wave of moisture had already passed to the central portion of the wick and was heading to the membrane end of the wick. The latter is supported by the finding that the impedance at the round window membrane end dropped during the first minute.

After 3 minutes, there is a notable cross-over in impedance signals between segments 1 and 5. For the first time, the application end of the wick becomes drier than the membrane end. This relationship is then maintained for the remainder of the experiment. The synchronous ascent of impedance signals from each segment and the absence of any further signal cross-over suggests that after the initial wicking period volume flows do not occur along the fluid saturated wick. Throughout the experiment the Microwick appears to hold more medication in its centre (particularly segments 3 and 4 compared to its extremities. The round window membrane is a semi-permeable membrane. It is known that drugs in direct contact with the membrane with a molecular weight of less than 1000, such as gentamicin, will diffuse across it. 
Table 2 Mean area with standard deviation for each sample before and after the Microwick. The percentage difference was also calculated

\begin{tabular}{|c|c|c|c|c|c|c|c|c|}
\hline Medication & $\begin{array}{l}\text { Mean Area } \\
\text { before } \\
\text { Microwick }\end{array}$ & $\begin{array}{l}\text { Std Dev } \\
\text { before } \\
\text { Microwick }\end{array}$ & \% Dev & $\begin{array}{l}\text { Mean } \\
\text { Area after } \\
\text { Microwick }\end{array}$ & $\begin{array}{l}\text { Std Dev } \\
\text { after } \\
\text { Microwick }\end{array}$ & $\%$ Dev & $\begin{array}{l}\text { Mean } \\
\text { Difference }\end{array}$ & $\begin{array}{l}\% \\
\text { Difference }\end{array}$ \\
\hline Garasone & $5.63 \mathrm{E}+10$ & $5.3 \mathrm{E}+09$ & 9.4 & $5.13 \mathrm{E}+10$ & $6.32 \mathrm{E}+09$ & 12.3 & $5.03 E+09$ & 8.936491 \\
\hline Cipro he & $4.85 \mathrm{E}+10$ & $3.83 \mathrm{E}+09$ & 7.9 & 4. $19 \mathrm{E}+10$ & $2.12 E+09$ & 5.1 & $6.66 \mathrm{E}+09$ & 13.73566 \\
\hline Dexamethasone & $1.94 \mathrm{E}+10$ & $1.31 E+09$ & 6.7 & $1.84 \mathrm{E}+10$ & $1.06 \mathrm{E}+09$ & 5.7 & $9.46 \mathrm{E}+08$ & 4.88279 \\
\hline Methylprednisolone & $6.93 \mathrm{E}+10$ & $2.62 \mathrm{E}+09$ & 3.8 & $1.56 \mathrm{E}+10$ & $9.85 E+08$ & 6.3 & $5.36 \mathrm{E}+10$ & 77.42593 \\
\hline SoluMedrol & $1.00 \mathrm{E}+1 \mathrm{I}$ & $7.22 \mathrm{E}+09$ & 7.2 & $9.83 \mathrm{E}+10$ & $4.78 \mathrm{E}+09$ & 4.9 & $2.2 \mathrm{E}+09$ & 2.186334 \\
\hline
\end{tabular}

Table 3 Viscosities with error calculations of five commonly prescribed inner ear medications

\begin{tabular}{|c|c|c|c|c|}
\hline Solutions & Viscosity (mPAS) & Normalized Values * & Mean & STD \\
\hline \multirow[t]{3}{*}{ Methylprednisolone Suspension } & 2.1 & 2.16 & 2.13 & 0.149 \\
\hline & 1.8 & 1.85 & & \\
\hline & 2.3 & 2.37 & & \\
\hline \multirow[t]{3}{*}{ Dexamethasone Injectable } & 0.963 & 0.78 & 0.81 & 0.059 \\
\hline & 0.971 & 0.787 & & \\
\hline & 1.064 & 0.862 & & \\
\hline \multirow[t]{3}{*}{ Dexamethasone Opthalmic Solution } & 1.044 & 0.846 & 0.77 & 0.085 \\
\hline & 0.912 & 0.739 & & \\
\hline & 0.896 & 0.726 & & \\
\hline \multirow[t]{3}{*}{ Garasone Opthalmic/Otic Solution } & 0.88 & 0.712 & 0.752 & 0.006 \\
\hline & 1.035 & 0.838 & & \\
\hline & 0.87 & 0.705 & & \\
\hline Distilled Water (CRC value) & 0.692 & & & \\
\hline
\end{tabular}

*Normalized Values:The viscometers were both calibrated with water at $37^{\circ} \mathrm{C}$ and compared to the CRC value for water.The CRC value for water was found to be $81 \%$ of the value determined by the Viscomat

As there is no subsequent driver for flow along the wick towards the rwm after initial saturation, it is important to establish how long this wicking process maintains wetness of the rwm end of the wick and in turn diffusion across the rwm. The whole of the Microwick is dry after approximately 105-110minutes. The ends dry first. The application end becomes completely dry after 90-95minutes. According to electrical impedance testing, the drug is only available to the rwm for 100-105minutes.

\section{Second experiment}

It was important to verify that the electrical impedance device was actually measuring "wetness". The weight tests were performed as our primary method of validation. Ideally the measurements for weight and resistance would have been taken simultaneously but logistics did not permit this. The time taken for the microwick to become dry in the impedance experiments and the weight tests were concordant (105-110minutes and 105-110minutes respectively). By taking the results of both experiments, we are able to demonstrate a significant linear, inverse relationship between weight and resistance. Consequently, we can be reasonably certain that the resistance device measures "wetness" and that our apparatus was functioning as intended. In order to determine the reliability of the measurements obtained from the device we adjusted a number of environmental conditions. Increasing humidity, adding a heat source and adding further drops after an hour influenced the drying times in a predictable manner. This confirms our device was performing correctly.

\section{Third experiment}

The endpoint of the electrical impedance tests was return of the impedance to the pre-test, dry state level (i.e. to $32 \mathrm{~K}$ ohms). The third experiment was conducted to verify this endpoint. The results suggest that availability of drug at the rwm end of the wick stops when the rwm end of the wick (segment 5) reaches an impedance of
$26 \mathrm{~K}$ ohms. One possible explanation for this discrepancy is that there is a small segment of the wick beyond the last electrode. Therefore, although segment 5 may be moist, the portion of the wick beyond this and in direct contact with rwm, would be dry. We therefore decided to go back to our electrical impedance data and use $26000 \mathrm{ohms}$ as our endpoint for drug availability at the rwm end of the microwick (segment 5). Consequently, drug is available to the rwm from the wick for 70-75minutes. The point at which no more drug is available to be delivered from the Microwick is the point at which, theoretically, more medication should be applied to the ear canal in order to achieve a more sustained release delivery profile to the round window membrane.

These results are preliminary and require further exploration, but as of now it can be hypothesized that if continuous flow of medication to the round window membrane was desired, about three drops would need to be applied by the patient every hour. Application of medication every hour is tedious and is not likely to be well accepted by patients. Therefore, further investigations into Microwick specifications (porosity, capillary action, surface tension etc) and Microwick coverings should be made to increase the drying time, which will increase the time between applications.

The results for individuals will also be heavily influenced by environmental temperature and humidity etc etc.

As allowed to above one of the major problems encountered with this experiment was evaporation particularly when working with such minute quantities of liquids. The filter paper was weighed immediately after the allotted time period in order to minimize this. Also, the mass of the filter paper changed over time according to changes in environmental conditions within the laboratory, or if the paper touched something moist such as an ungloved hand. Consequently, it was necessary to take mass measurements directly before and after 
using the paper. In addition, the filter paper had very different qualities compared to the round window membrane, which it was substituting so qualities concerning filtering effects, speed of diffusion, porosity, etc were not observed in this experiment.

\section{Fourth experiment}

Methylprednisolone showed a marked difference after being passed through the Microwick. About $80 \%$ of its components were retained by the Microwick. The other suspension analysed, Cipro HC, showed a $14 \%$ decrease. Cipro HC contains an emulsifier, which allows the particles in the suspension to behave like fruit floating in jello. The emulsifier keeps the drug particles floating evenly spaced which may help their progress through the Microwick. In comparison, Methylprednisolone does not contain an emulsifier and its particles therefore settle. This may be the reason why its components are retained in the Microwick. Consequently, suspensions do not appear to be the ideal medication to use through a Microwick as their drug components are retained by it. The differences for the remaining solutions were small and analogous to deviations measured within the solutions themselves, which implies that the Microwick does not act as a filter to any components of these drugs.

\section{Fifth experiment}

The application of viscosity to the project pertains to the flow rate of the various solutions used through the Microwick. Theoretically, a solution with lower viscosity will have an increased flow rate; therefore will be more suited for use through the Microwick. Methylprednisolone is a suspension and consequently was more difficult to test than the other samples. It was shaken immediately before testing but still had a higher standard deviation than the other solutions. Since it does not contain an emulsifier its particles are able to settle, which prevents the suspension from being homogenous and causes the viscosity and flow dynamics of the mixture to be sporadic. All of the solutions have a higher viscosity than water, specifically the Methylprednisolone. The two ophthalmic solutions, Dexamethasone and Garasone, have the same viscosity range.

\section{Technical issues}

Several technical issues were encountered during the assembly of the resistance device. The way the leads made contact with the Microwick was the most problematic issue. The prototype consisted of six metal plates placed side-by-side. The $0.5 \mathrm{~mm}$ space between each plate was filled with rubber insulation. A groove for the Microwick to lie was created along the center of the plates. Each plate was connected to a thin wire and the wires were fed to similar circuitry as in the working model. The major problem with this initial design was the fact that as the wet Microwick lay on the plates, some of the moisture was able to seep down through the apparatus to short the circuit or distort resistances. To overcome this, a model was built in which the Microwick was placed onto a series of six taut wires. Conceptually this arrangement would have been ideal, except that the thin wires necessary to measure the small Microwick were very fragile and bent easily, eliminating contact with the Microwick at certain points. Finally, the idea of approaching the Microwick from the top was developed. We were confident that no shorting would occur and that all leads were in equal contact with the Microwick.
The simulated ear canal environment also posed problems. It was difficult at first to keep the temperature and/or humidity constant and at desired levels. However, adjusting the dimmer switch on the light bulb and varying the temperature on the humidifier enabled relatively stable conditions to be maintained. Whilst conducting the Microwick experiments it became apparent that following the application of three drops to one end, it would become completely soaked, almost immediately. Although the custom-built electrical resistance device was able to measure a fall in resistance across all electrode pairs, it was not sensitive enough to detect the fast travelling wave of moisture sequentially during the initial wetting phase. Our model did not use a grommet sleeve, as it would have hampered electrode insertion into the tiny wick.

\section{Conclusion}

The Silverstein Microwick has been used successfully to treat patients with Ménière's disease. The inner ear drug concentrations and profiles that can be achieved with this device will be largely influenced by its wicking characteristics and the drug dosing schedule applied. Our study set out to examine specific properties of the Silverstein Microwick. The results are preliminary and require further investigation. Furthermore, only inner ear studies will be able to precisely profile and quantify the changes in drug concentration that occur beyond the RWM when using this device.

A two drop application of Garasone ${ }^{\circledR}$ onto the microwick sustains delivery to the RWM end for $80-85$ minutes. It follows that a typical dosing regimen of 3 drops of medication three times daily for Ménière's disease would deliver an interrupted flow of medication to the inner ear. Our data could be used to help design dosing protocols that impart more sustained or even continuous delivery to the RWM.

\section{Acknowledgments}

None.

\section{Conflicts of interest}

Author declares there are no conflicts of interest.

\section{Funding}

None.

\section{References}

1. Hoffer ME, Allen K, Kopke RD, et al. ranstympanic versus sustainedrelease administration of gentamicin: kinetics, morphology, and function. Laryngoscope. 2001;111(8):1343-1357.

2. Wanamaker HH. Perfusion of the inner ear: basic science considerations. Curr Opin Otolaryngol Head Neck Surg. 2001; (5):329-332.

3. Kimura R, Iverson N, Sothard R. Selective lesions of the vestibular labyrinth. Ann Otol Rhinol Laryngol. 1988;97(6 Pt 1):577-584.

4. Sheppard WM, Wanamaker HH, Pack A, et al.Direct round window application of gentamicin with varying delivery vehicles: $A$ comparison of ototoxicity. Otolaryngol Head Neck Surg. 2004;131(6):890-896.

5. Jackson LE, Silverstein H. Chemical perfusion of the inner ear Otolaryngol Clin North Am. 2002;35(3):639-653. 